\title{
Research on Learning Control and Decision of Foreign Language Based on Neural Network
}

\author{
Bing He \\ College of International Studies, Wuzhou University, Wuzhou, China \\ h_e_bing@126.com
}

\begin{abstract}
Keywords: neural network; double weighted neural network; algorithm of choosing nearest location; control and decision; transformation function
\end{abstract}

\begin{abstract}
The study of CET4 and CET6 is the important part of English learning, and it is helpful for the comprehensive application of English and the work after graduation, so the learning methods of CET4 and CET6 have got more and more attention of teachers and students. This paper was based on double weighted neural network, and it used the new algorithm of continuous speech recognition to construct the model of continuous speech recognition, in this way it can avoid the influence of speech's misidentification on the listening of CET4 and CET6. It was on the basis of fuzzy neural network to put forward the fast learning algorithm of choosing nearest location. This algorithm can improve the learning efficiency, and it is helpful for the study of reading comprehension of CET4 and CET6. Finally this paper put forward the methods of learning CET4 and CET6, and it provided the theoretical basis for the control and decision of CET4 and CET6.
\end{abstract}

\section{Introduction}

The learning of CET4 and CET6 is the significant part of English learning. The listening of CET4 and CET6 is the weak part of many students, and the low scores of listening made them not pass the exams of CET4 and CET6[1,2]. Many people think that listening is difficult, therefore they usually give up the opportunity to improve listening[3]. At the same time they want to improve the scores from other aspects, and this is a big misunderstanding. Reading comprehension is really hard to be improved, and the improvement of reading comprehension is not as easy as people's ideas[4,5]. So this article was based on double weighted neural network, and it used the new algorithm of continuous speech recognition to construct the model of continuous speech recognition, in this way it can avoid the speech's misidentification and improve the achievements of listening of CET4 and CET6[6]. According to the reading comprehension, this paper was on the basis of fuzzy neural network to put forward the fast learning algorithm of choosing nearest location, and it can improve the learning efficiency of reading comprehension.

\section{Basic structural theory of neural network}

A. Artificial neural model

Artificial neurons also can be called as neurons, and it is the basic unit of ANN. A neuron has $\mathrm{j}$ different inputs, and the number of same output nonlinear units is k. As shown in Figure 1, netj is the first $\mathrm{j}$ neurons, $\alpha \mathrm{j}$ is the threshold of the first $\mathrm{j}$ neuron, and $\mathrm{Bj}$ is the first $\mathrm{j}$ neuron output information. $\mathrm{A} 1, \ldots, \mathrm{Ai}, \ldots, \mathrm{An}$ are respectively the transferred information from the net $1, \ldots$ neti, ...netn to the first $\mathrm{j}$ neuron. $\mathrm{Cij}$ is the connection weight from neuron neti to netj. Obviously it has the following formula[7]: $B_{i}=p \sum\left(B_{i j} A_{i j}-\alpha j\right),(i=1,2, \ldots, n, j=1,2, \ldots, n)$

The $\mathrm{p}$ is the transfer function, and the common transfer functions are:

(1) Threshold type of transfer function $[8] \mathrm{p}($ net $)=\left\{\begin{array}{l}1, \text { net } \geq 0 \\ 0, \text { net } \geq \dot{0}\end{array}(2)\right.$

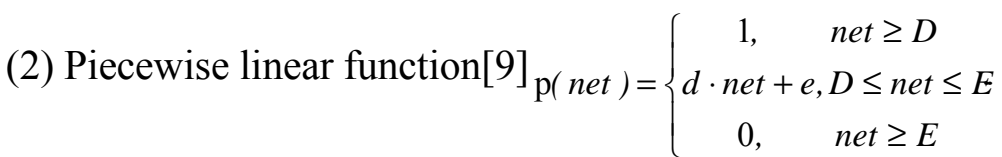


B. Artificial neural structure

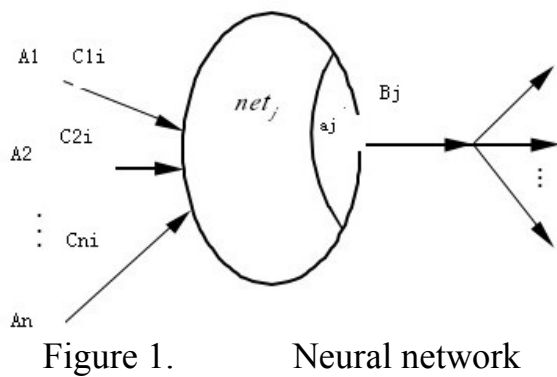

Neural network model includes single layer and multiple layers, and the earliest neural network models were generally single layer or two layers. The interconnection of every neuron is the single layer; all the neurons have mutual connection and single layer has local interconnection; the neurons in the same layer do not have connection; the neurons between the two layers have interconnection; the output layer and input layer have the feedback connection. In the neural network model, the structure of multilayer neural network is a typical form[10]. As shown in Figure 2, the weighted input signals of neurons are sent to the activity function through the accumulation to determine neurons' output. The neural network has the ability of learning, at the same time it has the structure of parallel processing.

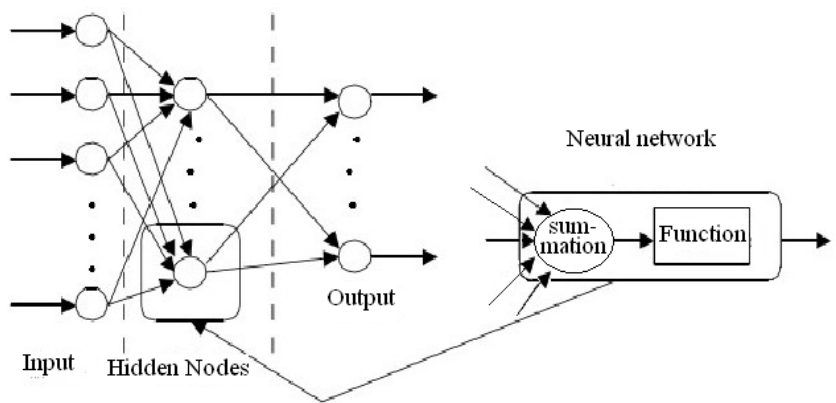

Figure 2. $\quad$ Multilayer model

\section{Control and decision of speech recognition neural network of CET4 and CET6}

The basic calculation formula of double weighted neural network is:

$$
B=f\left[\sum_{j=0}^{M}\left(\frac{C_{j}\left(A_{j}-C^{\prime}\right)}{\left|C_{j}\left(A_{j}-C^{\prime}\right)\right|}\right)\left|C_{j}\left(A_{j}-C_{j}^{\prime}\right)\right|^{p}-\alpha\right]
$$

$\mathrm{B}$ is the output of neuron, and $\mathrm{f}$ is the neurons incentive function. $C_{j}$ is threshold value of the neuron, $C_{j}^{\prime}$ is the first $\mathrm{j}$ core weights, and $\mathrm{s}$ is the method's parameter that can decide the plus or minus of the single item.

Figure 3. Final statistical results of recognition system
\begin{tabular}{|l|l|l|}
\hline \multicolumn{1}{|c|}{ Model } & Correct rate & Error rate \\
\hline Speech recognition of entire sentence & $84.62 \%$ & $15.38 \%$ \\
\hline Speech recognition of keywords & $93.5 \%$ & $6.5 \%$ \\
\hline
\end{tabular}

From the Table 1 we can know that the words' error rate of our recognition system finally reaches to $15.38 \%$, and the words' correct rate is $84.62 \%$. The whole sentence is limited by the grammar, so we can use the method of keywords recognition. It only needs to identify keywords in the whole sentence, and this can be thought that it identifies the sentence[11]. In our recognition system, the correct rate of entire sentences' speech recognition is $93.50 \%$. So in the listening of CET4 and CET6, we can't only pay attention to the whole sentence, and we should extract the keywords. The content of the keywords can infer the whole sentence, and this can enhance the correct rate of the listening recognition.

\section{Neural network's control and decision of fast learning methods of CET4 and CET6}

Reading comprehension is the key of CET4 and CET6, and the reading speed determines the efficiency[12]. We can use the learning method of choosing nearest location, and this method can be 
used to study the ways of improving the reading speed. We can use the following fuzzy statement to express the fuzzy model:

$$
\begin{aligned}
& P 1: \text { if } x \quad A 1 \text { and } y \quad B 1 \text { then } z \quad C 1 \\
& P 2 \text { : if } x i s A 2 \text { and } y \text { is } B 2 \text { then } z \text { is } C 2 \\
& \text { P3: if } x \text { An and } y \quad B n \text { then } z \quad C 3
\end{aligned}
$$

According the fuzzy model in the formula (5), the fuzzy sets of the $\mathrm{x}$ and $\mathrm{y}$ are:

$$
\begin{aligned}
& \left\{\begin{array}{ll}
A 1 & A 2
\end{array} A n \in P(X)\right. \\
& B 1 \quad B 2 \cdots B n \in P(Y)
\end{aligned}
$$

When the values of $\mathrm{x}$ and $\mathrm{y}$ are inputted, and any combination of $\mathrm{P}(\mathrm{x})$ and $\mathrm{P}(\mathrm{y})$ is:

$$
\operatorname{Dij}=T\{\mu(A i(x)), \mu(\mathrm{Bj}(\mathrm{y}))\}
$$

$\mu(\operatorname{Ai}(x)), \mu(\mathrm{Bj}(\mathrm{y}))$ are respectively the membership grades of the first $\mathrm{i}$ and the first $\mathrm{j}$ fuzzy sets in the theory field of $\mathrm{x}$ and $\mathrm{y}$, and $\mathrm{T}$ is the modal operator of $\mathrm{T}$.

Research of learning algorithm is an important direction of the neural network, and rapidity is an important indicator to measure the learning algorithm. According to characteristics of DIFNN, fuzzy neural network and fuzzy relation model, this paper was based on the conclusion of the fuzzy sets' operation to put forward a kind of fast learning algorithm[13]. The algorithm of choosing nearest location has its unique aspects, and it only has to learn the training once, so its efficiency is very high. In the algorithm, we also introduce the concept of confidence to improve the reliability of the study results. The practice shows that the algorithm of choosing nearest location is especially applicable to capture, express structure information of many variables. So in the tests of CET4 and CET6, we can use it as a method to answer the questions of reading comprehension, and it can greatly improve the efficiency of reading comprehension.

\section{Learning method of CET4 and CET6}

Poor listening mainly has several reasons.

(1) The aspect of pronunciation

The pronunciation is unqualified, and many people don't memory the pronunciation to remember the words. When they answer the listening questions, they don't know the words that they have heard, and their scores are naturally low. And some students don't have standard pronunciation, and this causes no reaction to the words in the listening section. So the students should pay attention to correct their pronunciation.

(2) The students don't adapt to the listening environment

The students can't adapt to the listening environment, this is the second reason of students' poor listening ability. In fact listening contents of CET4 and CET6 are frequent dialogues in the life and campus[14]. The students don't have to understand every word in the listening tests, and they can use the method of speech recognition to identify keywords in the sentences. According to the formula(4), we find the key point of information output to determine the important content of the listening tests.

(3) The cultural background and listening

The students are not familiar with the cultural background and common phrases, this is the third reason, and this causes the barriers of understanding the content of listening. A lot of colloquial vocabularies of oral English are not the relevant contents in the books, but they often appear in the listening tests.

Reading comprehension usually has two types: main idea questions and specific questions. The difficulty of the main idea questions is not big, and in the process of training the main ideas of the texts can be grasped. Generally speaking the first paragraph and the first sentence of each paragraph of the paper have implied more information of the main idea, so we don't need to pay attention to the details in the process of consideration, and we can generally make the correct answers after careful analysis[15]. The range of specific questions is very wide, some questions investigate the words or the meaning of sentences, and some questions investigate the detail aspects and the inferences. The error rate of inference questions is higher, but in fact the inferences need the most direct and the simplest vocabularies, and the meaning of sentence should combine the content of context, at the same it has certain requirements for the mastery of the vocabulary and sentences. The best way of reading is to go through the article and understand the main ideas of the text. We should understand the meaning of each paragraph, and then read all the questions. The specific questions can be positioned to the original article, and they are based on the analyses of the relevant statements to find out the answers. The environment needs to improve efficiency, according to the formula(7), We can 
use the fast learning algorithm of choosing nearest location. This can greatly improve the efficiency of reading comprehension and save a lot of time, and it provides the safeguard for the high scores of CET4 and CET6.

\section{Conclusion}

This paper was based on the theoretical method of double weighted neural network, and it used the new algorithm to construct the continuous speech recognition model. According to the researches, it is known that the identification of key words can replace the recognition of the full sentences in the listening of CET4 and CET6, and this can improve the accuracy of the listening. On the basis of fuzzy neural network, this paper put forward the fast learning algorithm of choosing nearest location. This method can improve the learning efficiency, and it is helpful for the reading comprehension of CET4 and CET6. Finally this paper put forward the learning method of CET4 and CET6, and it provided the theoretical basis for the control and decision of CET4 and CET6. The continuous speech segmentation is difficult, and it directly affects the recognition rate of speech recognition. The experiments can improve that if the errors are separated and modified in the earlier stage, the words' error rate of the system at least can be reduced by $11.7 \%$. This paper changed the traditional speech recognition, and it used the dynamic search algorithm to realize the speech recognition without segmentation. It started from the double weights complicated geometry and gave the learning algorithm of double weighted neural network in the speech recognition. In the future this algorithm can be applied to the speech recognition of the listening of CET4 and CET6. This paper also introduced the algorithm of choosing nearest location, it is especially applicable to capture, express the structure information of many variables. In the tests of CET4 and CET6, we can use it as a method to answer the questions of reading comprehension, and it can greatly improve the efficiency of reading comprehension.

\section{References}

[1] Jianmin Li. Research on Chinese speech recognition.Tsinghua master's thesis, 2010:36-39.

[2] Shoujue Wang, Jian Xu, Xianbao Wang. Research on more lens of face identification system based on bionic pattern recognition .Journal of electronics, 2010(07):19-22.

[3] Zhaozhou Li, Xiangdong Chen. Discussion on basic mathematical model of general neural network hardware .Journal of electronics, 2011(05): 77-80.

[4] Gang Ding, Cengke Zhang, Cengjin Han. Adaptive fuzzy control of nonlinear system robust .Journal of automation, 2010(09):56-62.

[5] Tianping Zhang, Chunbo Feng. Adaptive fuzzy sliding mode control of the nonlinear system .ACTA AUTOMATICA SINICA, 2009(04): 61-63.

[6] Tianping Zhang. Design and analysis of adaptive fuzzy sliding mode controller .ACTA AUTOMATICA SINICA, 2010(08):70-73.

[7] Tianping Zhang. New design of adaptive fuzzy variable structure controller of .Control and decision making, 2011(10):59-62.

[8] Lixin Wang. Adaptive fuzzy system and control - analysis of design and stability .Beijing: national defense industry press, 2009(05):24-26.

[9] Jun Zhou. Design and stability analysis of nonlinear fuzzy indirect and direct adaptive controller .Control and decision making, 2011(3):93-96

[10] Xudong Feng, Chen Fang. Application of neural network in the diagnosis of plant diseases and insect pests .Computer development and application, 2010(11):31-34.

[11] Han Xia. Practical guide of MATLAB neural network toolbox .Internet downloads data, 2010(08):12-15.

[12] Junrang Ju, Rong Zhuo. The convenient realization of BP neural network in MATLAB .Xinjiang petroleum institute report, 2011(06):47- 50.

[13] Hongxing Zheng, Jihuan Yao. MATLAB5. X kit use skills and instance . Huazhong press, 2010:105-108.

[14] Shuntian Lou, Shi Yang. Analysis and design based on the MATLAB system . Xian of electronic science and technology press, 2012(05):29-31.

[15] Ming Zhou, Changhong Li, Humin Lei. Technology of MATLAB graphics .Northwest industry press, 2011:159-168. 